\title{
Preoperative Tissue Doppler Imaging-Derived Atrial Conduction Time Predicts Postoperative Atrial Fibrillation in Patients Undergoing Mitral Valve Surgery for Mitral Valve Regurgitation
}

\author{
Shinya Takahashi, MD, PhD; Keijiro Katayama, MD, PhD; Masazumi Watanabe, MD; \\ Hiroshi Kodama, MD; Takahiro Taguchi, MD; Tatsuya Kurosaki, MD, PhD; \\ Katsuhiko Imai, MD, PhD; Taijiro Sueda, MD, PhD
}

\begin{abstract}
Background: Postoperative atrial fibrillation (POAF) is a common complication of cardiac surgery and may result in stroke, heart failure and poor prognosis. This study evaluated a novel index of total atrial conduction time derived from the P-wave onset (lead II) to the peak A' wave on tissue Doppler imaging (PA-TDI duration) in patients undergoing mitral valve surgery (MVS) for mitral valve regurgitation.
\end{abstract}

Methods and Results: Seventy-three patients undergoing MVS had transthoracic echocardiography with tissue Doppler imaging preoperatively and were monitored postoperatively with continuous electrocardiographic telemetry for 14 days. Preoperative characteristics, echocardiographic data, operative data and postoperative findings were compared between patients with $(n=44)$ and without $(n=29)$ POAF. Postoperative cardiac events were higher in patients with than without POAF $(12 / 44,27 \%$ vs. $3 / 29,10 \% ; P=0.0798)$ and cerebral events occurred in only 2 POAF patients. On multivariate analysis the independent predictors of POAF were degenerative disease etiology (OR, 4.61; 95\% Cl: 1.41-15.0; $\mathrm{P}=0.0112$ ) and PA-TDI duration (OR, 1.04; 95\% Cl: 1.01-1.07; $\mathrm{P}=0.0048)$. On ROC curve analysis a PA-TDI cut-off of $159.4 \mathrm{~ms}$ was optimal for predicting POAF.

Conclusions: PA-TDI duration was an independent predictor of POAF after MVS. Patients with PA-TDI duration $>159.4 \mathrm{~ms}$ should be considered high risk and treated appropriately to improve outcome. (Circ J 2016; 80: $101-109)$

Key Words: Atrial fibrillation; Mitral valve regurgitation; Mitral valve surgery; Total atrial conduction time

$\mathbf{P}$ ostoperative atrial fibrillation (POAF) is a common complication after cardiac surgery, occurring in $10-65 \%$ of patients. ${ }^{1}$ Several studies have shown that POAF is associated with longer hospital stay, higher risk of stroke, permanent pacemaker implantation, and increased inhospital and long-term mortality. ${ }^{2}$ The incidence of PAOF was higher after mitral valve surgery (MVS) than after coronary artery bypass grafting (CABG) or aortic valve surgery. ${ }^{3}$ This indicates that POAF should be prevented and controlled postoperatively, but most previous studies have reported the incidence of POAF after CABG and aortic valve surgery. Thus, there are limited data on the risk of POAF after MVS. ${ }^{3}$

\section{Editorial p 58}

Many clinical variables, including preoperative characteris- tics, comorbidities, intraoperative findings and postoperative complications and requirements have been evaluated as predictors of POAF, but only advanced age was a consistent predictor among studeis. ${ }^{4}$ Several studies achieved much better prediction of POAF by measuring left atrial (LA) characteristics such as LA volume index (LAVI), LA strain and total atrial conduction time (TACT). TACT is theoretically measured on electrocardiography (ECG), but there are several alternative methods for measuring TACT. The time interval from P-wave onset on ECG to the peak of the A' lateral wave on tissue Doppler imaging (TDI; PA-TDI duration) provides a reliable estimation of TACT, and PA-TDI duration has been shown to be significantly related to new-onset AF. ${ }^{5,6}$ Our previous studies showed that PA-TDI duration was predictive of $\mathrm{AF}$ in patients undergoing off-pump CABG and aortic valve surgery. ${ }^{7,8}$ The present study therefore evaluate the efficacy of

Received July 15, 2015; revised manuscript received September 17, 2015; accepted October 4, 2015; released online November 4, 2015

Time for primary review: 23 days

Department of Cardiovascular Surgery, Hiroshima University Hospital, Hiroshima, Japan

Mailing address: Shinya Takahashi, MD, PhD, Department of Cardiovascular Surgery, Hiroshima University Hospital, 1-2-3 Kasumi,

Minami-ku, Hiroshima 734-8551, Japan. E-mail: shiny@mte.biglobe.ne.jp

ISSN-1346-9843 doi:10.1253/circj.CJ-15-0796

All rights are reserved to the Japanese Circulation Society. For permissions, please e-mail: cj@j-circ.or.jp 


\begin{tabular}{|c|c|c|c|}
\hline Variable & No POAF ( $n=29)$ & POAF $(n=44)$ & P-value \\
\hline Age (years) & $59.4 \pm 16.6$ & $65.8 \pm 11.3$ & 0.0520 \\
\hline Gender (male) & $17(59)$ & $32(73)$ & 0.2093 \\
\hline $\mathrm{BMI}\left(\mathrm{kg} / \mathrm{m}^{2}\right)$ & $21.5 \pm 3.8$ & $21.8 \pm 2.8$ & 0.7004 \\
\hline BSA $\left(m^{2}\right)$ (Dubois method) & $1.59 \pm 0.22$ & $1.60 \pm 0.17$ & 0.7595 \\
\hline Hypertension & $15(52)$ & $23(52)$ & 0.9634 \\
\hline Hyperlipidemia & $6(21)$ & $17(39)$ & 0.1063 \\
\hline Diabetes & $3(10)$ & $10(34)$ & 0.1760 \\
\hline HbA1c (\%) (NGSP) & $5.8 \pm 1.3$ & $5.7 \pm 0.6$ & 0.6087 \\
\hline COPD & $3(10)$ & $2(5)$ & 0.3371 \\
\hline Creatinine $(\mathrm{mg} / \mathrm{dl})$ & $1.39 \pm 2.10$ & $1.15 \pm 1.02$ & 0.5129 \\
\hline Hemodialysis & $2(7)$ & $2(5)$ & 0.6658 \\
\hline \multicolumn{4}{|l|}{ Medications } \\
\hline ACEI/ARB & $15(52)$ & $23(52)$ & 0.9634 \\
\hline Calcium channel blocker & $7(24)$ & $8(18)$ & 0.5377 \\
\hline$\beta$-blocker & $8(28)$ & $13(30)$ & 0.8564 \\
\hline Statin & $6(21)$ & $9(20)$ & 0.9806 \\
\hline History of PAF & $7(24)$ & $11(25)$ & 0.9334 \\
\hline \multicolumn{4}{|l|}{ Etiology } \\
\hline Degenerative & $14(48)$ & $36(82)$ & 0.0045 \\
\hline Functional & $5(17)$ & $5(11)$ & \\
\hline Infective & $10(34)$ & $3(7)$ & \\
\hline Duration of MR (years) & $0.9 \pm 2.4$ & $3.0 \pm 4.0$ & 0.0134 \\
\hline NT-proBNP (pg/ml) & $1,209.7 \pm 1,602.7$ & $3,575.3 \pm 11,956.4$ & 0.3303 \\
\hline $\mathrm{CRP}(\mathrm{mg} / \mathrm{dl})$ & $1.14 \pm 1.95$ & $0.39 \pm 0.78$ & 0.0242 \\
\hline
\end{tabular}

Data given as mean \pm SD or $n(\%)$. ACEI, angiotensin-converting enzyme inhibitor; ARB, angiotensin-II receptor blocker; BMI, body mass index; BSA, body surface area; COPD, chronic obstructive pulmonary disease; CRP, C-reactive protein; MR, mitral valve regurgitation; MVS, mitral valve surgery; NGSP, National Glycohemoglobin Standardization Program; NT-proBNP, N-terminal pro-B-type natriuretic peptide; PAF, paroxysmal atrial fibrillation; $\mathrm{POAF}$, postoperative atrial fibrillation.

PA-TDI duration for predicting POAF in patients undergoing MVS for mitral valve regurgitation (MR).

\section{Methods}

\section{Subjects}

Seventy-three consecutive patients with MR, who underwent MVS at Hiroshima University Hospital between January 2009 and December 2014, were retrospectively enrolled. The exclusion criteria were as follows: chronic AF, emergency surgical procedure, prior implantation of a permanent pacemaker or implantable cardioverter defibrillator, and prior resynchronization therapy. Based on these criteria, 55 patients were excluded, including 37 patients with chronic AF.

The institutional review board approved the use of patient data in this retrospective study.

All patients had 12-lead ECG, transthoracic echocardiography and coronary angiography before surgery. All patients were monitored with continuous ECG telemetry for $\geq 2$ weeks following MVS. A patient was determined to have AF when any episode lasted $>5 \mathrm{~min}$. Cardiologists in the cardiovascular intensive care unit, who were not informed of the study, confirmed the diagnosis of $\mathrm{AF}$ and initiated prompt treatment using $\beta$-blockers, calcium antagonists, anti-arrhythmic drugs and/or defibrillation. Patients were divided into 2 groups according to the presence of POAF. The etiology of mitral valve disease was divided into 3 groups based on the modified classification of Carpentier: degenerative, functional, and infective. ${ }^{9}$ In this series, all patients with degenerative disease had chordae rupture with/without secondary leaflet changes. Patients with functional valve disease included 8 cases of ischemic mitral regurgitation and 1 case of dilated cardiomyopathy-related MR. Infective mitral valve disease was defined based on a history of acute or subacute infective endocarditis and mitral valve disease with or without vegetation, which required surgical repair. Infective endocarditis may complicate pre-existing valve disease, but completely healed and chronic mitral valve disease was included in the degenerative disease category. ${ }^{10}$ Duration of MR was defined as from the diagnosis of MR on echocardiography to the date of operation. Postoperative cardiac failure was defined as the onset of symptoms and signs including general fatigue, respiratory disorder, systemic edema and pleural effusion secondary to abnormal cardiac function requiring additional use of catecholamines, vasodilators, diuretics and/or mechanical support. Patient characteristics, ECG and echocardiographic parameters, medications and perioperative parameters were compared between the groups with and without POAF.

\section{Echocardiography}

Transthoracic echocardiography was performed as described previously. ${ }^{8}$ In brief, left ventricular (LV) diameter, wall thickness, mitral valve inflow pattern (E wave, A wave, E wave deceleration time, E/A ratio), LV ejection fraction, LA volumes and LAVI, LV mass and MR severity were determined on 2-D, M-mode, pulsed wave, continuous wave, color 


\begin{tabular}{|c|c|c|c|}
\hline Variables & No POAF ( $n=29)$ & POAF $(n=44)$ & P-value \\
\hline \multicolumn{4}{|l|}{ Electrocardiography } \\
\hline P-wave duration (ms) & $115.6 \pm 15.1$ & $118.8 \pm 12.6$ & 0.3295 \\
\hline \multicolumn{4}{|l|}{ Echocardiography } \\
\hline LA dimension (mm) & $44.0 \pm 7.6$ & $43.6 \pm 6.4$ & 0.8033 \\
\hline LV end-diastolic dimension (mm) & $55.7 \pm 8.2$ & $56.3 \pm 7.3$ & 0.7646 \\
\hline LV end-systolic dimension (mm) & $37.1 \pm 9.9$ & $38.4 \pm 9.2$ & 0.5896 \\
\hline Interventricular septal thickness (mm) & $9.2 \pm 1.5$ & $9.1 \pm 1.0$ & 0.6600 \\
\hline Posterior wall thickness (mm) & $9.2 \pm 1.6$ & $9.0 \pm 1.2$ & 0.5927 \\
\hline E wave $(\mathrm{cm} / \mathrm{s})$ & $113.7 \pm 43.9$ & $105.5 \pm 31.5$ & 0.3524 \\
\hline A wave $(\mathrm{cm} / \mathrm{s})$ & $80.9 \pm 39.2$ & $63.6 \pm 25.1$ & 0.0273 \\
\hline $\mathrm{E} / \mathrm{A}$ ratio & $1.7 \pm 1.0$ & $1.8 \pm 0.8$ & 0.3824 \\
\hline Deceleration time (ms) & $202.5 \pm 59.3$ & $207.8 \pm 77.6$ & 0.7607 \\
\hline LV ejection fraction (\%) & $62.6 \pm 14.7$ & $58.2 \pm 13.7$ & 0.1983 \\
\hline LA volume $(\mathrm{ml})$ & $86.0 \pm 26.1$ & $93.7 \pm 32.7$ & 0.3198 \\
\hline LA volume index $\left(\mathrm{ml} / \mathrm{m}^{2}\right)$ & $53.4 \pm 16.2$ & $58.7 \pm 20.6$ & 0.2767 \\
\hline LV end-diastolic volume (ml) & $135.8 \pm 51.5$ & $136.6 \pm 47.7$ & 0.9431 \\
\hline LV end-diastolic volume index $\left(\mathrm{ml} / \mathrm{m}^{2}\right)$ & $85.4 \pm 27.9$ & $84.9 \pm 29.0$ & 0.9409 \\
\hline LV end-systolic volume (ml) & $52.1 \pm 35.7$ & $60.9 \pm 36.6$ & 0.3272 \\
\hline LV end-systolic volume index $\left(\mathrm{ml} / \mathrm{m}^{2}\right)$ & $32.6 \pm 20.6$ & $37.9 \pm 22.7$ & 0.3397 \\
\hline LV mass $(\mathrm{g})$ & $234.1 \pm 69.1$ & $234.1 \pm 66.6$ & 0.9968 \\
\hline LV mass index $\left(\mathrm{g} / \mathrm{m}^{2}\right)$ & $147.4 \pm 37.5$ & $146.7 \pm 41.0$ & 0.9419 \\
\hline \multicolumn{4}{|l|}{ MR grade } \\
\hline 2 & $2(7)$ & $3(7)$ & 0.3276 \\
\hline 3 & $5(17)$ & $14(32)$ & \\
\hline 4 & $22(76)$ & $27(61)$ & \\
\hline \multicolumn{4}{|l|}{ TR grade } \\
\hline 0 & $1(3)$ & $7(16)$ & 0.1691 \\
\hline 1 & $23(79)$ & $27(61)$ & \\
\hline 2 & $25(17)$ & $10(23)$ & \\
\hline TR PG max (mmHg) & $38.6 \pm 19.1$ & $33.9 \pm 12.1$ & 0.2477 \\
\hline$A^{\prime}$ wave peak $(\mathrm{cm} / \mathrm{s})$ & $8.8 \pm 3.3$ & $8.9 \pm 3.4$ & 0.9256 \\
\hline e' wave peak $(\mathrm{cm} / \mathrm{s})$ & $10.1 \pm 4.2$ & $9.1 \pm 3.5$ & 0.3131 \\
\hline E/e' ratio (septal) & $16.6 \pm 7.2$ & $18.5 \pm 9.4$ & 0.3729 \\
\hline E/e' ratio (lateral) & $12.4 \pm 8.0$ & $13.4 \pm 7.2$ & 0.5739 \\
\hline PA-TDI duration (ms) & $140.6 \pm 20.1$ & $159.3 \pm 21.5$ & 0.0006 \\
\hline
\end{tabular}

Data given as mean \pm SD or $n(\%)$. ECG, electrocardiography; LA, left atrial; LV, left ventricular; PA-TDI duration, time interval from $P$-wave onset on ECG to the peak of the A' lateral wave on tissue Doppler imaging; $P G$, pressure gradient; TR, tricuspid valve regurgitation. Other abbreviations as in Table 1.

flow and TDI. TDI was performed with transducer frequencies of $3.5-4.0 \mathrm{MHz}$ by adjusting the spectral pulsed Doppler signal filters to acquire the Nyquist limit of $15-20 \mathrm{~cm} / \mathrm{s}$ and using the minimal optimal gain. Spectral pulsed Doppler was used to measure myocardial TDI velocities (early diastolic and late diastolic velocities) for the LV lateral wall from the apical 4-chamber view. PA-TDI interval was defined as the time interval from the P-wave onset (lead II) to the peak of the A' wave on the tissue Doppler tracing of the LA lateral wall, ${ }^{7}$ and was measured in 3 cardiac cycles and averaged. Evaluation of transthoracic echocardiography was performed $<1$ month before surgery and $<1$ month after surgery. Experienced echocardiographers (blinded to the clinical information) conducted all echocardiography and analyzed the results.

\section{Surgical Technique and Postoperative Management}

All preoperative cardiac medications, including $\beta$-blockers, calcium channel antagonists, angiotensin-converting enzyme inhibitors and angiotensin receptor blockers (except for nonsteroidal anti-inflammatory drugs), were continued until 1 day before surgery. The patients underwent MVS using standard surgical techniques. General anesthesia was induced and maintained with i.v. infusion of fentanyl and propofol. Muscle relaxation was achieved with pancuronium. Through a median sternotomy, cardiopulmonary bypass was established by ascending aortic and bicaval right atrial cannulae. Cardiac arrest was obtained via antegrade blood cardioplegia followed by intermittent retrograde cardioplegia. The diseased mitral valve was examined through a right-sided left atriotomy. In 46 cases, mitral valve repair was performed with standard techniques including triangle/quadrangular resection and suture, artificial neochordae reconstruction, and edge-to-edge and ring annuloplasty. Mitral valve replacement was performed in 27 patients, involving a bioprosthetic valve in 17 and a mechanical valve in 10. In patients with a history of paroxysmal AF, either maze procedure $(n=10)$ or pulmonary vein orifice isolation (PVOI, 
$\mathrm{n}=6$ ) was performed at the discretion of the surgeon using a radiofrequency isolator (Atricure, $\mathrm{OH}$, USA), and the orifice of the left appendage was closed with a 4-0 polypropylene running suture. All patients were observed in the intensive care unit and woke up from anesthesia approximately $2 \mathrm{~h}$ after the operation or on the following morning. Pulmonary capillary wedge pressure (PCWP) and central venous pressure (CVP) were measured every morning with a pulmonary artery catheter connected to a fluid-filled transducer. The transducer was balanced before the study with the zero level at the midaxillary line. An anesthesiologist blinded to the echocardiographic data carried out the pressure measurements. Each pressure was measured at the end of expiration and averaged over 6 respiratory cycles.

The total volume administered (volume-in) and eliminated via all routes (volume-out) were recorded, and net fluid balance (NFB) was calculated by subtracting the total volume-out from the volume-in during the operation, on the day after surgery, and on the first and second postoperative day (POD). The patients were transferred to a general ward on the 2nd POD. Anticoagulation with heparin was started on the 1st POD followed by warfarin therapy within 4 days after the operation. Prothrombin time (international normalized ratio) was controlled between 2.0 and 2.5 .

\section{Statistical Analysis}

Data are presented as mean \pm SD. Continuous variables were compared between groups using Student's t-test. Categorical variables were compared using chi-squared test. The relationship between 2 continuous variables was analyzed using Pearson's correlation. Variables with $\mathrm{P}<0.1$ on Student's t-test or chi-squared test were entered into univariate and multivariate logistic regression analysis to identify the predictors of POAF, and OR and 95\% CI were calculated. Receiver operating characteristic (ROC) curves were calculated to determine optimal cut-offs for predicting POAF. All statistical analysis were performed using SPSS version 22.0 (SPSS, Chicago, IL, USA) and $\mathrm{R}$ version 3.1.0. $\mathrm{P}<0.05$ was considered significant.

\section{Results}

\section{Preoperative Patient Characteristics}

The overall incidence of POAF was 60\% (44/73 patients). Onset of POAF was at 4.8 \pm 3.1 POD. In 25 POAF patients $(57 \%)$, POAF occurred on the 2 nd or 5 th POD, and the incidence gradually decreased by the 14th POD. In 2 patients (3\%), AF persisted at discharge and 1 received catheter ablation resulting in sinus rhythm. Preoperative characteristics are listed in Table 1. Patients with POAF had a relatively higher age than those without POAF $(65.8 \pm 11.3$ vs. $59.4 \pm 16.6$ years old, $\mathrm{P}=0.0520$ ), lower $\mathrm{C}$-reactive protein (CRP; $0.39 \pm 0.78$ vs. $1.14 \pm 1.95 \mathrm{mg} / \mathrm{dl}, \mathrm{P}=0.0242$ ) and longer duration of $\mathrm{MR}$

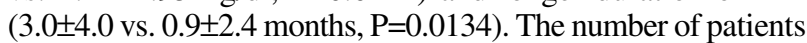
with degenerative disease in the POAF group was larger than the number with infective disease $(\mathrm{P}=0.0027)$ and the number with functional disease $(\mathrm{P}=0.0079)$. The other preoperative characteristics were similar between the 2 groups.

\section{Preoperative ECG and Echocardiography}

There was no significant difference in $\mathrm{P}$-wave duration between the patients with and without POAF on ECG, whereas the A wave and PA-TDI were significantly different between the 2 groups on echocardiography (Table 2). The degree of MR was not associated with the incidence of POAF.

\section{Perioperative and Postoperative Factors}

There were no significant differences in intraoperative factors between the 2 groups (Table 3 ). Intraoperative and postoperative medications, including the use of catecholamines, $\beta$-blockers and carperitide, were not related to the incidence of POAF. Preoperative CVP was lower in patients with than without POAF ( $4.9 \pm 1.9$ vs. $6.2 \pm 2.8 \mathrm{mmHg}, \mathrm{P}=0.0230)$. There were no significant differences in postoperative PCWP, postoperative CVP and NFB between patients with and without POAF.

There was also no significant difference in the duration of hospital stay between patients with and without POAF. In patients with degenerative mitral valve disease, however, hospital stay was relatively longer in those with than without POAF (29.2 \pm 19.1 days vs. $20.2 \pm 5.5, \mathrm{P}=0.0911$ ), and the number of patients with hospital stay $>27$ days was significantly higher in those with than without POAF $(16 / 36,44 \%$ vs. $1 / 14$, $8 \%, \mathrm{P}=0.0183)$. The number of patients with postoperative cardiac failure was slightly higher in those with than without POAF $(12 / 44,27 \%$ vs. $3 / 29,10 \%, \mathrm{P}=0.0798)$. The causes of cardiac failure in the POAF group included recurrent and longstanding POAF, AF with bradycardia and low cardiac function. Patients who had heart failure required prolonged i.v. catecholamine infusion. POAF in most of these patients was controlled with $\beta$-blocker. Permanent pacemaker implantation for sick sinus syndrome in 1 patient in the POAF group prolonged the hospital stay. In 2 patients, sick sinus syndrome worsened at 5 and 7 months after operation, and permanent pacemakers were implanted. Two patients had AF at discharge, one of whom was treated with catheter ablation resulting in sinus rhythm, while the other had AF controlled with $\beta$-blocker and amiodarone.

There was no hospital death in this series. Two patients with POAF had cerebral infarction that occurred during POAF on POD 12 and on POD 50 immediately after discharge and were transferred to the hospital for intensive rehabilitation. No patients in the study had perioperative myocardial infarction during hospitalization, but 1 patient who underwent MVS and CABG required percutaneous coronary intervention due to a residual ischemic lesion in the left anterior descending artery after unexpected bypass to the first diagonal branch.

\section{Predictors of POAF}

Results of univariate and multivariate analyses are listed in Table 3. On univariate analysis degenerative disease, duration of MR, A wave peak velocity, PA-TDI duration and preoperative CVP were significant predictors of POAF.

A positive correlation was seen between $\mathrm{P}$-wave duration and PA-TDI duration $(\mathrm{r}=0.4440 ; \mathrm{P}=0.0001)$ and between PATDI duration and age $(\mathrm{r}=0.3981 ; \mathrm{P}=0.0007)$. CRP was significantly lower in patients with degenerative disease than in those with functional and infective disease $(0.26 \pm 0.55$ vs. $0.74 \pm 1.17$ vs. $2.30 \pm 2.45 \mathrm{mg} / \mathrm{dl}, \mathrm{P}=0.0466$ degenerative vs. functional, $\mathrm{P}<0.0001$ degenerative vs. infective). The duration of MR was significantly longer in degenerative than infective disease ( $2.18 \pm 2.03$ vs. $0.37 \pm 0.53$ years, $\mathrm{P}=0.0468$ ). Moreover, a modest correlation was seen between CRP and preoperative CVP ( $\mathrm{r}=0.2945 ; \mathrm{P}=0.0114)$ and between CRP and preoperative PCWP ( $\mathrm{r}=0.3405 ; \mathrm{P}=0.0032)$.

Table 4 lists comparison of the preoperative and postoperative P-wave duration, amplitude and morphology, and the PA-TDI duration between patients with or without maze/ PVOI procedure. In patients undergoing maze/PVOI, there was no significant difference between preoperative and postoperative $\mathrm{P}$-wave duration, but postoperative $\mathrm{P}$-wave ampli- 


\begin{tabular}{|c|c|c|c|}
\hline Variable & No POAF $(n=29)$ & POAF $(n=44)$ & P-value \\
\hline \multicolumn{4}{|l|}{ Preoperative data } \\
\hline CVP (mmHg) & $6.2 \pm 2.8$ & $4.9 \pm 1.9$ & 0.0230 \\
\hline PCWP (mmHg) & $12.5 \pm 6.8$ & $9.8 \pm 4.7$ & 0.0426 \\
\hline \multicolumn{4}{|l|}{ Intraoperative findings } \\
\hline \multicolumn{4}{|l|}{ Type of repair } \\
\hline Bioprosthesis & $7(24)$ & $10(23)$ & 0.3327 \\
\hline Mechanical & $6(21)$ & $4(9)$ & \\
\hline Plasty & $16(55)$ & $30(68)$ & \\
\hline Isolated mitral valve surgery & $15(52)$ & $24(55)$ & 0.8131 \\
\hline Tricuspid annuloplasty & $1(3)$ & $8(25)$ & 0.0610 \\
\hline Maze procedure & $6(21)$ & $10(23)$ & 0.8368 \\
\hline Operation time (min) & $308.6 \pm 76.4$ & $310.0 \pm 105.1$ & 0.9519 \\
\hline ECC time $(\min )$ & $150.0 \pm 36.6$ & $186.8 \pm 202.9$ & 0.3377 \\
\hline ACC time (min) & $103.9 \pm 35.8$ & $96.9 \pm 30.9$ & 0.3813 \\
\hline Bleeding (ml) & $1,111.0 \pm 821.5$ & $1,060.1 \pm 748.5$ & 0.7853 \\
\hline \multicolumn{4}{|l|}{ Intraoperative medications } \\
\hline Noradrenaline & $5(17)$ & $5(11)$ & 0.4748 \\
\hline Dopamine & $28(97)$ & $43(98)$ & 0.7634 \\
\hline Dobutamine & $8(28)$ & $12(27)$ & 0.9766 \\
\hline Milislol & $14(48)$ & $15(34)$ & 0.2255 \\
\hline Landiolol & $1(3)$ & $5(11)$ & 0.2282 \\
\hline Nicardipine & $17(59)$ & $32(73)$ & 0.2093 \\
\hline Carperitide & $16(55)$ & $30(68)$ & 0.2599 \\
\hline \multicolumn{4}{|l|}{ Net fluid balance } \\
\hline Intraoperative (ml) & $488.8 \pm 1,220.0$ & $548.9 \pm 1,296.2$ & 0.8432 \\
\hline POD 0 & $24.9 \pm 573.9$ & $88.6 \pm 1,024.4$ & 0.7622 \\
\hline POD 1 & $380.0 \pm 730.0$ & $88.7 \pm 1,014.1$ & 0.1863 \\
\hline POD 2 & $-895.7 \pm 766.3$ & $-1,261.6 \pm 947.8$ & 0.0867 \\
\hline \multicolumn{4}{|l|}{ Postoperative findings } \\
\hline \multicolumn{4}{|l|}{ Postoperative medications } \\
\hline Dopamine & $21(72)$ & $29(66)$ & 0.5584 \\
\hline Dobutamine & $10(34)$ & $14(32)$ & 0.8125 \\
\hline Milislol & $11(38)$ & $14(32)$ & 0.5902 \\
\hline Nicardipine & $4(14)$ & $7(16)$ & 0.8047 \\
\hline Carperitide & $17(59)$ & $19(43)$ & 0.1967 \\
\hline \multicolumn{4}{|l|}{ Oral medications } \\
\hline$\beta$-blocker & $9(31)$ & $16(36)$ & 0.6387 \\
\hline Amiodarone & $2(7)$ & $3(68)$ & 0.9897 \\
\hline Maximum PCWP (mmHg) & $19.5 \pm 3.6$ & $20.3 \pm 5.0$ & 0.4243 \\
\hline Maximum CVP $(\mathrm{mmHg})$ & $10.8 \pm 2.6$ & $10.4 \pm 3.3$ & 0.5485 \\
\hline Temporary pacing & $18(62)$ & $35(80)$ & 0.0680 \\
\hline Cardiac failure & $3(10)$ & $12(27)$ & 0.0798 \\
\hline Cerebral infarction & 0 & $2(5)$ & 0.2444 \\
\hline Hospital stay (day) & $26.5 \pm 10.1$ & $25.1 \pm 8.0$ & 0.6337 \\
\hline P-wave duration (ms) & $111.3 \pm 14.5$ & $110.4 \pm 16.8$ & 0.8120 \\
\hline PA-TDI duration (ms) & $144.0 \pm 21.4$ & $157.4 \pm 21.7$ & 0.0238 \\
\hline
\end{tabular}

Data given as mean \pm SD or $n(\%)$. ACC, aortic cross-clamp time; CVP, central venous pressure; ECC extracorporeal time; PCWP, pulmonary capillary wedge pressure; POD, postoperative day. Other abbreviations as in Table 1.

tude was significantly smaller than the preoperative amplitude, and the number of patients with positive-negative morphology in lead II was significantly increased. There were no significant differences between preoperative and postoperative PA-TDI. Preoperative PA-TDI was relatively longer in patients with than without maze/PVOI (160.8 \pm 28.6 vs.
149.6 $\pm 20.3 \mathrm{~ms}, \mathrm{P}=0.0838$ ), but there was no significant difference in postoperative PA-TDI between patients with or without maze/PVOI (159.9 \pm 26.8 vs. $150.1 \pm 23.3, \mathrm{P}=0.2671)$.

Table 5 lists univariate and multivariate analysis of risk factors for predicting POAF. On multivariate analysis degenerative disease (OR, 4.61; 95\% CI: $1.41-15.0 ; \mathrm{P}=0.0112)$ and 


\begin{tabular}{|c|c|c|c|}
\hline Variables & Preoperative & Postoperative & P-value \\
\hline \multicolumn{4}{|l|}{ P-wave duration (ms) } \\
\hline Maze/PVOI $(n=16)$ & $117.6 \pm 17.5$ & $115.1 \pm 12.7$ & 0.8869 \\
\hline No maze/PVOI $(n=57)$ & $117.5 \pm 12.5$ & $109.6 \pm 16.4$ & 0.0007 \\
\hline \multicolumn{4}{|c|}{ P-wave amplitude in lead II $(\mu \mathrm{V})$} \\
\hline Maze/PVOI & $112.1 \pm 57.6$ & $56.3 \pm 27.4$ & 0.0005 \\
\hline No maze/PVOI & $84.6 \pm 36.2$ & $72.2 \pm 31.7$ & 0.1032 \\
\hline \multicolumn{4}{|c|}{ P-wave morphology in lead II } \\
\hline \multicolumn{4}{|c|}{ Maze/PVOI } \\
\hline Positive & $16(100)$ & $11(69)$ & 0.0311 \\
\hline,+- & 0 & $5(31)$ & \\
\hline \multicolumn{4}{|l|}{ No maze/PVOI } \\
\hline Positive & $55(96)$ & $52(91)$ & 0.4382 \\
\hline,+- & $2(4)$ & $5(9)$ & \\
\hline \multicolumn{4}{|l|}{ PA-TDI duration (ms) } \\
\hline Maze/PVOI & $160.8 \pm 28.6$ & $159.9 \pm 26.8$ & 0.8625 \\
\hline No maze/PVOI & $149.6 \pm 20.3$ & $150.1 \pm 23.3$ & 0.5535 \\
\hline
\end{tabular}

Data given as mean \pm SD or $n(\%)$. (+,-), positive-negative morphology; PVOI, pulmonary vein orifice isolation. Other abbreviation as in Table 2.

PA-TDI duration (OR, 1.04; 95\% CI: 1.01-1.07; $\mathrm{P}=0.0048$ ) were significant independent predictors of POAF.

ROC curve analysis showed that the area under the curve for PA-TDI duration was 0.737 (95\% CI: 0.62-0.86; P<0.0001; Figure). The optimal PA-TDI cut-off duration was $159.4 \mathrm{~ms}$ (sensitivity, 55.8\%; specificity, 84.6\%).

\section{Discussion}

This study has shown that preoperative PA-DTI duration predicted POAF in patients who underwent MVS for MR without chronic AF.

POAF is one of the most common complications after cardiac surgery and contributes to the worsening of the postoperative state and prognosis, and increases hospital costs. ${ }^{11} \mathrm{~A}$ multicenter study in patients undergoing cardiac surgery showed that mitral valve replacement was a risk factor for POAF, and prevalence of morbidities including hospital death, 6-month mortality and stroke was significantly higher in patients with than without POAF. ${ }^{3}$ Melby et al reported that patients receiving MVS had a higher risk of POAF compared with patients receiving CABG. ${ }^{12}$ The present study showed that the incidence of postoperative heart failure tended to be higher in patients with POAF, and 2 patients had cerebral infarction during POAF. Furthermore, in patients with degenerative disease, the presence of POAF significantly prolonged hospital stay by more than 27 days. Preoperative assessment and preventive management for POAF would be beneficial for improving prognosis in these patients.

Many risk factors for the development of POAF have been identified. The preoperative risk factors include older age, male gender, previous history of AF, LV failure, LA enlargement, chronic obstructive pulmonary disease, diabetes mellitus, obesity and reoperation. The perioperative and postoperative risk factors include intraoperative and postoperative catecholamine use, respiratory failure and postoperative LV diastolic dysfunction. ${ }^{13,14}$ Many LA characteristics such as LA size, LA volume, LA area, LA ejection fraction, LA strain and TACT have been show to predict POAF, as briefly described in our previous articles. ${ }^{8,13}$

LA enlargement is known to be a risk factor for POAF, as reported in several previous studies. ${ }^{15}$ In the present study, there was no significant difference in LAVI between patients with and without POAF, but LAVI in both groups was larger than that in previous reports, and both groups in the present study had an increased risk of POAF, $3,7,8$

TACT is defined as the time between the initial and the last portion of the atrial myocardium to depolarize, which usually reflects the interval from the sinus node to LA wall activation, and this was discussed in our previous reports. ${ }^{5,7,8}$ Theoretically, TACT is measured by the duration of the $\mathrm{P}$ wave on 12-lead surface ECG, but there are several reports that measured TACT on echocardiography. ${ }^{7,8}$ The PA-TDI duration used in the present study was described by Merckx et al and was measured only in tissue Doppler mode on echocardiography. ${ }^{5}$ They also mentioned that PA-TDI duration included the time for propagation of the impulse to the LA as well as the time required for mechanical coupling of the LA; therefore, the PA-TDI interval overestimates TACT. PA-TDI duration reflects both electrical and structural remodeling in the atria. ${ }^{16}$ Electrical remodeling has been known to appear at an earlier phase than structural remodeling. ${ }^{17,18}$ In the present study, PATDI duration was significantly longer than $\mathrm{P}$-wave duration (152.4 \pm 22.9 vs. $117.6 \pm 13.7 \mathrm{~ms}, \mathrm{P}<0.0001)$.

Several reports have shown that PA-TDI is associated with the occurrence of AF. de Vos et al showed that prolonged PATDI duration was the most important predictor of new-onset AF. ${ }^{6}$ Another report suggested that prolonged PA-TDI duration was associated with the recurrence of $\mathrm{AF}$ after catheter ablation. ${ }^{16}$ Recently, Özlü et al showed that LA maximum volume and PA-TDI duration were independent predictors of POAF after conventional CABG. ${ }^{19}$ Our previous studies also showed that PA-TDI duration was an independent predictor of POAF in patients undergoing off-pump CABG and aortic valve surgery. 7,8

The aim of the present study was to determine the optimal cut-off of PA-TDI duration to predict POAF in patients with MR. In an outpatient clinical study, de Vos et al showed that 


\begin{tabular}{lccc}
\hline $\begin{array}{l}\text { Table 5. Independent Predictors of POAF } \\
\quad \text { Variables }\end{array}$ & OR & $\mathbf{9 5 \%} \mathbf{~ I ~}$ & P-value \\
Univariate & & & \\
Age & 1.04 & $0.999-1.07$ & 0.0580 \\
Etiology: degenerative & 4.82 & $1.68-13.9$ & 0.0035 \\
Duration of MR & 1.37 & $1.03-1.83$ & 0.0318 \\
CRP & 0.64 & $0.40-1.002$ & 0.0511 \\
A wave (cm/s) & 0.98 & $0.97-0.999$ & 0.0387 \\
PA-TDI duration (ms) & 1.04 & $1.02-1.07$ & 0.0020 \\
Preoperative CVP & 0.78 & $0.62-0.98$ & 0.0320 \\
Preoperative PCWP & 0.92 & $0.84-1.002$ & 0.0541 \\
Tricuspid annuloplasty & 6.22 & $0.73-52.7$ & 0.0936 \\
Multivariate & & & 0.0112 \\
Etiology: degenerative & 4.61 & $1.41-15.0$ & 0.0048 \\
PA-TDI duration (ms) & 1.04 & $1.01-1.07$ & \\
\hline
\end{tabular}

Abbreviations as in Tables 1-3.

patients with cardiac disease and PA-TDI duration $>165 \mathrm{~ms}$ had a reasonable chance of developing AF. ${ }^{6}$ In our previous study in patients undergoing off-pump $\mathrm{CABG}$ and aortic valve surgery, the optimal PA-TDI duration cut-offs for predicting POAF were 141 and $147.3 \mathrm{~ms}$, respectively. ${ }^{7,8}$ In the present study, we found that the optimal cut-off for PA-TDI duration was $>159.4 \mathrm{~ms}$. The difference in optimal PA-TDI cut-off may be related to the size of the LA and degree of surgical manipulation, and thus the optimal cut-off should be evaluated separately.

The large variability in patient demographic factors including etiology of mitral disease and concomitant cardiac disease affects the correlation of several factors. The present study included patients with endocarditis, which was usually diagnosed at the point of admission, and they required preoperative antibacterial therapy and surgery. Therefore, in patients with infective disease, the duration of MR was relatively short and CRP was elevated. In the present study, however, data were not sufficient to determine how etiology might have influenced the correlation between CRP and preoperative PCWP and CVP.

The $\mathrm{E}$ wave, $\mathrm{A}$ wave and $\mathrm{E} / \mathrm{A}$ ratio are affected by cardiac conditions including LA and LV size and function, heart rate, and preload and afterload; thus, they must be measured accurately. ${ }^{20}$ The mechanisms responsible for changes in these variables accurately in disease states remain undefined, but in patients undergoing $\mathrm{CABG}, \mathrm{A}$ wave velocity was significantly shorter in those with than without POAF. ${ }^{21}$ Decreased A wave velocity in patients with POAF may be associated with depression of atrial contractility. ${ }^{22}$ These findings are similar to the present findings and may support the present results, but further investigation is required.

Given that $\mathrm{P}$-wave morphology reflects electrical activation pattern, it may be modified by maze procedure, PVOI, and catheter ablation. ${ }^{23,24}$ The $\mathrm{P}$-wave duration recorded on signalaveraged ECG in patients undergoing MVS with maze procedure for chronic AF was longer than that in patients with normal sinus rhythm preoperatively $(150 \pm 20$ vs. $122 \pm 11){ }^{25}$ The average $\mathrm{P}$-wave amplitude in lead II after maze/PVOI was $<0.06 \pm 0.02 \mathrm{mV}$ and this was relatively small. ${ }^{23}$ The $\mathrm{P}$ wave in lead II showed positive-negative morphology in 59\% (89/150). ${ }^{24}$ These morphological features of the $\mathrm{P}$ wave were obtained when chronic AF was restored to sinus rhythm and were seen only after the operation. In the present study, P-wave ampli-

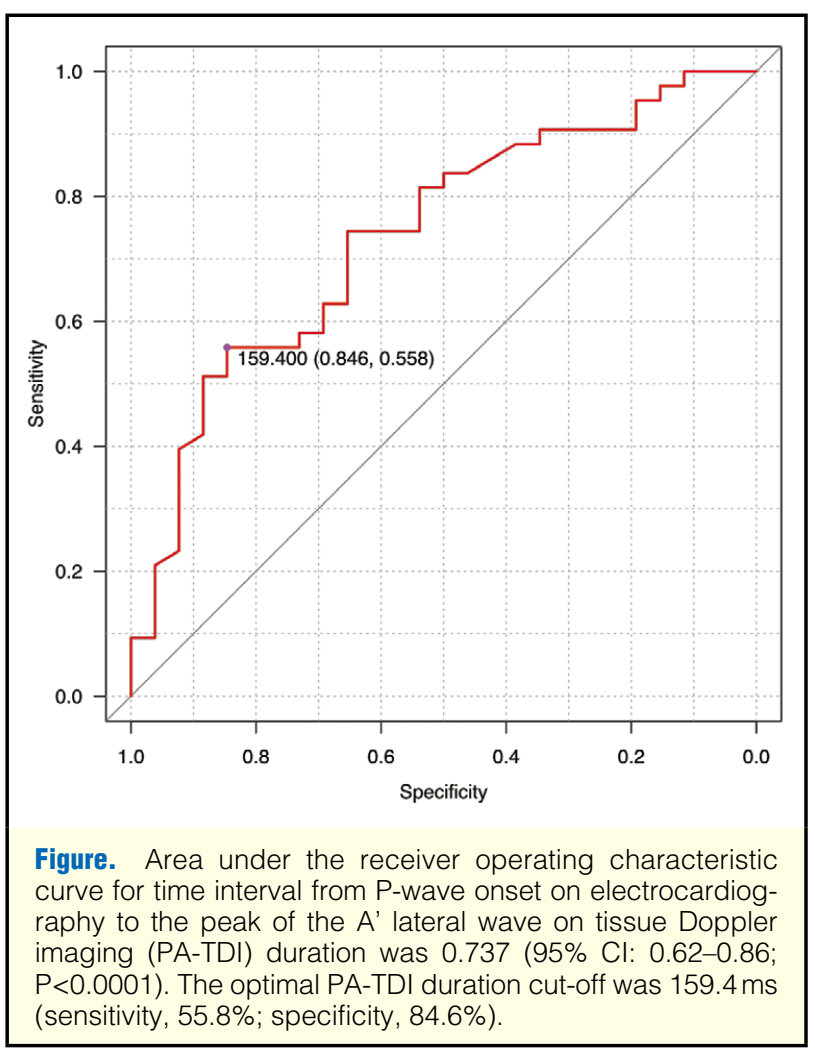

tude in patients undergoing maze/PVOI significantly decreased after operation, and there was a significant change in P-wave polarity. There was no significant difference between the preoperative and postoperative PA-TDI duration in patients with or without maze/PVOI. These results imply that maze/PVOI isolated the AF focus and decreased the total amount of activated atrium, but did not interfere with intra-atrial conduction. PA-TDI duration was relatively longer in patients with than without maze/PVOI, and this may be related to the small number of patients. P-wave duration measured on 12-lead ECG may underestimate TACT, especially when the P-wave is small, and this should be investigated using other methods including 
signal-averaged ECG.

Paroxysmal AF has been effectively prevented by maze and PVOI procedures. ${ }^{26,27}$ POAF and atrial tachycardia, however, were frequently observed after AF surgery. ${ }^{27}$ Although there was bias in the present study given that maze and PVOI procedures were performed only in patients with a documented history of paroxysmal AF, these procedures did not prevent POAF.

Prevention of POAF has been achieved with several therapeutic modalities including $\beta$-blockers, sotalol, amiodarone, angiotensin-II receptor antagonists and temporary pacing, but there is little evidence that these modalities can prevent POAF after valve surgery. ${ }^{28,29}$ One of the current strategies to prevent POAF in cardiac surgery is the prophylactic use of $\beta$-blockers. In the present study, oral $\beta$-blockers were used in 24 patients $(33 \%)$, and $\beta$-blocker use was not associated with the incidence of POAF. In some cases, $\beta$-blocker was not started immediately after operation. There were several reasons for this including sinus bradycardia and heart failure requiring catecholamine infusion, which should be considered a separate problem after MVS for MR. The efficacy of amiodarone for POAF was reported at a dose $\geq 400 \mathrm{mg} /$ day. ${ }^{30}$ Because the prevalence of amiodarone-induced pulmonary toxicity is estimated to be $5-15 \%$ in patients on $400 \mathrm{mg}$ /day and $0.1-0.5 \%$ in patients on $\leq 200 \mathrm{mg} /$ day, amiodarone should be used with careful observation. ${ }^{31}$

\section{Study Limitations}

The present study has several limitations. First, we examined a small number of patients in a single institution, and the study design was retrospective without randomization. The present findings should be validated in a larger prospective study. Second, this study included patients who underwent MVS for $\mathrm{MR}$, and most patients had multiple diseases including paroxysmal AF, coronary artery disease, and aortic valve disease that required concomitant procedures, which may have increased the risk of POAF. Only 39 (54\%) patients, however, underwent isolated MVS, and it was not possible to completely exclude patients with concomitant diseases.

In this study, the incidence of POAF was higher than that in previous studies. One reason may be that the definition of $\mathrm{AF}$ was shorter ( $5 \mathrm{~min}$ ) and differed from that used in other studies. Patients were monitored on continuous ECG telemetry for $\geq 2$ weeks, and POAF occurred at 8-14 days after operation in 9 cases. The definition and monitoring period may account for the higher POAF incidence in the present study.

Our current strategy to prevent POAF is to treat patients with a conventional strategy if PA-TDI duration is $<159.4 \mathrm{~ms}$ and/or the etiology is not degenerative disease, given that these patients have a low risk of POAF. If PA-TDI duration is $>159.4 \mathrm{~ms}$ and/or the etiology is degenerative disease, however, patients are at high risk for POAF and are treated with prophylactic $\beta$-blockers with or without amiodarone. I.v. randiolol is started during or after the operation and is followed by oral bisoprolol or carvedilol, starting at a low dose that is increased up to the maximum dose. In patients with uncontrolled POAF, oral amiodarone $<200 \mathrm{mg} /$ day is administered. Drugs are chosen according to patient comorbidities and ECG findings.

\section{Conclusions}

Increased PA-TDI duration was an independent predictor of POAF after MVS for MR. PA-TDI is easily measured, and patients with PA-TDI duration $>159.4 \mathrm{~ms}$ might be considered at high risk for POAF. These patients should be treated in order to reduce stroke and heart failure, shorten hospital stay and improve outcome.

\section{References}

1. Maisel WH, Rawn JD, Stevenson WG. Atrial fibrillation after cardiac surgery. Ann Intern Med 2001; 135: 1061-1073.

2. Echahidi N, Pibarot P, O'Hara G, Mathieu P. Mechanisms, prevention, and treatment of atrial fibrillation after cardiac surgery. $J \mathrm{Am}$ Coll Cardiol 2008; 51: 793-801.

3. Almassi GH, Schowalter T, Nicolosi AC, Aggarwal A, Moritz TE, Henderson WG, et al. Atrial fibrillation after cardiac surgery: A major morbid event? Ann Surg 1997; 226: 501-511.

4. Kaireviciute D, Aidietis A, Lip GY. Atrial fibrillation following cardiac surgery: Clinical features and preventative strategies. Eur Heart J 2009; 30: 410-425.

5. Merckx KL, De Vos CB, Palmans A, Habets J, Cheriex EC, Crijns $\mathrm{HJ}$, et al. Atrial activation time determined by transthoracic Doppler tissue imaging can be used as an estimate of the total duration of atrial electrical activation. J Am Soc Echocardiogr 2005; 18: 940944.

6. de Vos CB, Weijs G, Crijns HJGM, Cheriex EC, Palmans A, Habets $\mathrm{J}$, et al. Atrial tissue Doppler imaging for prediction of new onset atrial fibrillation. Heart 2009; 95: 835-840.

7. Fujiwara M, Nakano Y, Hidaka T, Oda N, Uchimura Y, Sairaku A, et al. Prediction of atrial fibrillation after off-pump coronary artery bypass grafting using preoperative total atrial conduction time determined on tissue Doppler imaging. Circ J 2014; 78: 345-352.

8. Takahashi S, Fujiwara M, Watadani K, Taguchi T, Katayama K, Takasaki T, et al. Preoperative tissue Doppler imaging-derived atrial conduction time can predict postoperative atrial fibrillation in patients undergoing aortic valve replacement for aortic valve stenosis. Circ J 2014; 78: 2173-2181.

9. Carpentier A. Cardiac valve surgery: The "French correction". $J$ Thorac Cardiovasc Surg 1983; 86: 323-337.

10. Adams DH, Rosenhek R, Falk V. Degenerative mitral valve regurgitation: Best practice revolution. Eur Heart J 2010; 31: 1958-1966.

11. Mahoney EM, Thompson TD, Veledar E, Williams J, Weintraub WS. Cost-effectiveness of targeting patients undergoing cardiac surgery for therapy with intravenous amiodarone to prevent atrial fibrillation. J Am Coll Cardiol 2002; 40: 737-745.

12. Melby SJ, George JF, Picone DJ, Wallace JP, Davies JE, George DJ, et al. A time-related parametric risk factor analysis for postoperative atrial fibrillation after heart surgery. J Thorac Cardiovasc Surg 2015; 149: $886-892$.

13. Banach M, Goch A, Misztal M, Rysz J, Jaszewski R, Goch JH. Predictors of paroxysmal atrial fibrillation in patients undergoing aortic valve replacement. J Thorac Cardiovasc Surg 2007; 134: $1569-1576$.

14. Handa N, Miyata H, Motomura N, Nishina T, Takamoto S; Japan Adult Cardiovascular Database Organization. Procedure- and agespecific risk stratification of single aortic valve replacement in elderly patients based on Japan Adult Cardiovascular Surgery Database. Circ J 2012; 76: 356-364.

15. Osranek M, Fatema K, Qaddoura F, Al-Saileek A, Barnes ME, Bailey KR, et al. Left atrial volume predicts the risk of atrial fibrillation after cardiac surgery: A prospective study. J Am Coll Cardiol 2006; 48: 779-786.

16. Uijl DW, Gawrysiak M, Tops LF, Trines SA, Zeppenfeld K, Schalij $\mathrm{MJ}$, et al. Prognostic value of total atrial conduction time estimated with tissue Doppler imaging to predict the recurrence of atrial fibrillation after radiofrequency catheter ablation. Europace 2011; 13: $1533-1540$.

17. Ausma J, Velden HM, Lenders MH, Ankeren EP, Jongsma HJ, Ramaekers FC, et al. Reverse structural and gap-junctional remodeling after prolonged atrial fibrillation in the goat. Circulation 2003; 107: $2051-2058$.

18. Teh AW, Kistler PM, Lee G, Medi C, Heck PM, Spence SJ, et al. Electroanatomic remodeling of the left atrium in paroxysmal and persistent atrial fibrillation patients without structural heart disease. J Cardiovasc Electrophysiol 2012; 23: 232-238.

19. Özlü MF, Erdem K, Kırıș G, Parlar Aİ, Demirhan A, Ayhan SS, et al. Predictive value of total atrial conduction time measured with tissue Doppler imaging for postoperative atrial fibrillation after coronary artery bypass surgery. J Interv Card Electrophysiol 2013; 37: $27-33$.

20. Nishimura RA, Abel MD, Hatle LK, Tajik AJ. Assessment of diastolic function of the heart: Background and current applications of 
Doppler echocardiography, part II: Clinical studies. Mayo Clin Proc 1989; 64: 181-204.

21. Roshanali F, Mandegar MH, Yousefnia MA, Rayatzadeh H, Alaeddini F, Amouzadeh F. Prediction of atrial fibrillation via atrial electromechanical interval after coronary artery bypass grafting. Circulation 2007; 116: 2012-2017.

22. Barbier P, Alioto G, Guazzi MD. Left atrial function and ventricular filling in hypertensive patients with paroxysmal atrial fibrillation. $J$ Am Coll Cardiol 1994; 24: 165-170.

23. Park HE, Kim KH, Kim KB, Ahn H, Choi YS, Oh S. Characteristics of $\mathrm{P}$ wave in patients with sinus rhythm after maze operation. $J$ Korean Med Sci 2010; 25: 712-715.

24. Buber J, Luria D, Sternik L, Kuperstein R, Grupper A, Goldenberg I, et al. Morphological features of the P-waves at surface electrocardiogram as surrogate to mechanical function of the left atrium following a successful modified maze procedure. Europace 2014; 16: $578-586$.

25. Kamata J, Nakai K, Chiba N, Hosokawa S, Sato Y, Nasu M, et al Electrocardiographic nature of restored sinus rhythm after Cox maze procedure in patients with chronic atrial fibrillation who also had other cardiac surgery. Heart 1997; 77: 50-55.

26. Shimizu W, Kosakai Y, Inagaki M, Kurita T, Suyama K, Aihara N, et al. Electrophysiologic changes in arrhythmogenic substrate following the maze procedure in patients with lone and paroxysmal atrial fibrillation. Jpn Circ J 1997; 61: 988-996.

27. Gillinov AM, McCarthy PM, Blackstone EH, Rajeswaran J, Pettersson G, Sabik JF, et al. Surgical ablation of atrial fibrillation with bipolar radiofrequency as the primary modality. J Thorac Cardiovasc Surg 2005; 129: 1322-1329.

28. Arsenault KA, Yusuf AM, Crystal E, Healey JS, Morillo CA, Nair $\mathrm{GM}$, et al. Interventions for preventing post-operative atrial fibrillation in patients undergoing heart surgery. Cochrane Database Syst Rev 2013; (1): CD003611, doi:10.1002/14651858.

29. Crystal E, Connolly SJ, Sleik K, Ginger TJ, Yusuf S. Interventions on prevention of postoperative atrial fibrillation in patients undergoing heart surgery: A meta-analysis. Circulation 2002; 106: 75-80.

30. Giri S, White CM, Dunn AB, Felton K, Freeman-Bosco L, Reddy P, et al. Oral amiodarone for prevention of atrial fibrillation after open heart surgery, the Atrial Fibrillation Suppression Trial (AFIST): A randomised placebo-controlled trial. Lancet 2001; 357: 830-836.

31. Ott MC, Khoor A, Leventhal JP, Paterick TE, Burger CD. Pulmonary toxicity inpatients receiving low-dose amiodarone. Chest 2003; 123: $646-651$. 